\title{
Armazenando registros de colaboração utilizando triple store
}

\author{
João Toss Molon ${ }^{1}$, João Luis Tavares da Silva ${ }^{1}$, Tiago Thompsen Primo ${ }^{2}$, Elisa Boff ${ }^{1}$ \\ ${ }^{1}$ Centro de Computação e Tecnologia da Informação \\ Universidade de Caxias do Sul (UCS) - Caxias do Sul - RS - Brasil \\ ${ }^{2}$ Instituto de Informática - Universidade Federal do Rio Grande do Sul (UFRGS) \\ Caixa Postal 15.064 - 91.501-970 - Porto Alegre - RS - Brazil \\ \{jtmolon, joaoluis.tavares, tiagoprimo\}@gmail.com, eboff@ucs.br
}

\begin{abstract}
This paper's goal is the development of a prototype for the storage of Collaboration Registers in triple store data bases. On Communities of Practice context, Collaboration Registers can be seen as properties sets which detail the interactions that happen between community's members, distinct communities a their contents. Such registers are structured using the RDF data model. This prototype development is based on the Framework CoP. The storage of Collaboration Registers was proposed through triple store in a way to provide richer querys in a Communities of Practice context.
\end{abstract}

Resumo. Este artigo tem como objetivo o desenvolvimento de um protótipo para o armazenamento de Registros de Colaboração em bases de dados triple store. No contexto de Comunidades de Prática, Registros de Colaboração podem ser vistos como conjuntos de propriedades que detalham as interações que podem ocorrer entre os membros de uma comunidade, comunidades distintas e os seus conteúdos. Tais registros são estruturados utilizando-se o modelo RDF. $O$ desenvolvimento desse protótipo é efetuado com base no Framework CoP. O armazenamento dos Registros de Colaboração foi proposto através de uma triple store de maneira a proporcionar consultas mais ricas dentro do contexto de Comunidades de Prática.

\section{Introdução}

Através do uso de Tecnologias de Informação e Comunicação (TIC) na educação, a aprendizagem vem ganhando novas dimensões. Desde a exploração do computador para reprodução de modelos conhecidos até o uso de sistemas inteligentes para fins de ensino [Carbonell 1970]. Com o advento da Internet, de um lado, foram desenvolvidos no meio acadêmico os Ambientes Virtuais de Aprendizagem (AVA), enquanto no meio corporativo foram criados ambientes virtuais para o suporte a aprendizagem organizacional, as Comunidades de Prática (CoPs - Communities of Practice).

Com o surgimento de tecnologias para suporte à redes sociais, a aprendizagem social tem ganhado destaque nos desafios da educação. A aprendizagem social envolve grupos dinâmicos de pessoas que compartilham objetivos comuns e práticas sobre o conteúdo em questão. Este artigo parte do pressuposto que aprendizagem colaborativa [Bandura 1977, Hart 2011] constitui a arquitetura base das CoP segundo 
[Fiorio et al. 2011]. Neste sentido, a aprendizagem social é construída a partir de conversas e participação em uma CoP, e tem mais relação com a forma como se aprende do que com o conteúdo aprendido [Healy 2009]. Neste contexto, os "aprendizes sociais" assumem papéis dinâmicos, tanto como consumidores quanto de produtores de conhecimento, cujas contribuições estão relacionadas a suas habilidades, interesses e conhecimento.

Em [Fiorio et al. 2011] e [Ribeiro et al. 2011] propõe-se um Framework de Comunidades de Prática. Esta ferramenta tem o intuito de proporcionar uma plataforma de construção de comunidades virtuais, visando o desenvolvimento de práticas e habilidades relacionadas a um domínio específico.

O Framework CoP é constituído por vários componentes que oferecem funcionalidades de cadastro, autenticação, criação e gestão de conteúdo, voltados para o contexto de Comunidades de Prática. Estes componentes interagem a partir de vários núcleos de conhecimento: Domínio de Interesses, Perfis e Registros de Colaboração. O principal foco deste artigo encontra-se no núcleo de conhecimentos dos Registros de Colaboração, em que é preciso descrever em termos ontológicos as possíveis relações entre as características e interesses dos usuários através das suas interações em um ambiente dinâmico e distribuído. O Registro de Colaboração prevê conjuntos de propriedades que descrevem as formas de relacionamentos que podem ocorrer entre indivíduos, comunidades e seus conteúdos, através de um modelo ontológico [Ribeiro et al. 2011], [Primo et al. 2012].

Este artigo tem como principal objetivo o desenvolvimento de uma solução para armazenamento de dados de colaboração em bases de dados de triplas, aderente ao modelo de web semântica. A Seção 2 trata dos conceitos de Web Semântica, triple store, e do Framework de Comunidades de Prática. Na Seção 3 é apresentado o modelo arquitetural dos Registros de Colaboração. A Seção 4 apresenta o desenvolvimento já efetuado no protótipo, enquanto a Seção 5 apresenta as conclusões e trabalhos futuros.

\section{Revisão bibliográfica}

\subsection{Web Semântica}

O termo Web Semântica refere-se a rede de informações que dá significado aos conteúdos na Internet. Para [Berners-Lee et al. 2001], a Web Semântica pode ser vista como "uma extensão da Web atual, onde a informação tem um significado bem definido, permitindo que computadores e pessoas trabalhem em cooperação". Ou seja, além do conteúdo em si, que pode ser interpretado pelo ser humano, existem mais informações que permitem que os conteúdos sejam reconhecidos e manipulados de maneira mais significativa pelas máquinas. Tais informações permitem que os computadores executem tarefas mais sofisticadas e retornem resultados mais precisos para o usuário [Berners-Lee et al. 2001, Berners-Lee et al. 2006].

O primeiro passo para que isso ocorra é a estruturação dos dados. A utilização de linguagens e padrões apropriados para representação de um conteúdo permite que a sua estrutura, suas propriedades e suas relações com outros conteúdos tornem-se explícitas, facilitando e expandindo a gama de operações que os computadores podem processar sobre esses dados [Bizer et al. 2009]. Para isso, existem tecnologias específicas que 
desempenham esse papel. O XML (Extensible Markup Language) permite a criação de tags diversas, o que possibilita a estruturação da informação presente no documento. Porém, isso não é o bastante do ponto de vista semântico [Berners-Lee et al. 2001].

Para a adição de significado aos conteúdos da Web é utilizado o padrão RDF (Resource Description Framework). Esta tecnologia permite a representação de dados no modelo de um grafo, onde os registros são estruturados em triplas, cada uma contendo sujeito, predicado e objeto, representando uma sentença completa. Para a criação de vocabulários para definição da nomenclatura específica para um conjunto de dados de um determinado domínio utiliza-se o RDFS (RDF Schema). Pode-se dizer que "o RDFS é uma extensão semântica do RDF” [Brickley and Guha 2004].

\subsection{Triple Store}

Com o aumento da utilização de tecnologias como o RDF e o RDFS, surgiu a necessidade de criação de repositórios capazes de fazer a persistência de tais tipos de dados. São os denominados triple stores. Essas ferramentas possuem funcionalidades que permitem o armazenamento e acesso das triplas RDF [Berners-Lee et al. 2006]. E têm como principal aspecto a capacidade de fazer inferências a partir de consultas. A consulta a essas bases é feita através da linguagem SPARQL, que é é "uma linguagem de correspondência de grafos" [Perez et al. 2006].

Os triple stores diferenciam-se dos bancos relacionais, em primeiro lugar, pelo tipo de informação que é armazenada (triplas RDF nos triple stores e tabelas de dados nos relacionais) [Sequeda 2013]. Outro ponto em que os triple stores diferem dos bancos relacionais é em relação à modelagem da base de dados. Enquanto bancos relacionais necessitam de um schema previamente definido, os bancos de triplas são dinâmicos em relação à sua estrutura, com novos predicados podendo ser adicionados automaticamente. Também não é necessário estabelecer relações entre entidades previamente, uma vez que as essas ligações são feitas de forma direta [Aasman 2011].

A principal motivação para o uso de triple stores vem do fato dos mesmos serem mais aderentes ao modelo de Web Semântica, uma vez que são projetados com base no modelo de triplas. Ou seja, os dados são armazenados de acordo com o formato de grafos do RDF, eliminando a necessidade de mapeamentos para outros formatos [Faye et al. 2012].

\subsection{Framework CoP}

O Framework de Comunidades Prática proposto por [Fiorio et al. 2011] e [Ribeiro et al. 2011] disponibiliza um conjunto de ferramentas e componentes com funções de cadastro, autenticação, criação e gestão de conteúdo, todos voltados para o cenário de Comunidades de Prática. A Figura 1 representa o diagrama do framework, com base em uma ontologia de referência, representando seus componentes e as relações entre eles.

O foco do presente artigo está na classe Collaboration Registers (Registros de Colaboração). Essa classe descreve as relações que podem ser estabelecidas entre aspectos e interesses de usuários através das interações destes em um ambiente dinâmico e distribuído. É através de conjuntos de propriedades que são descritas formas de 


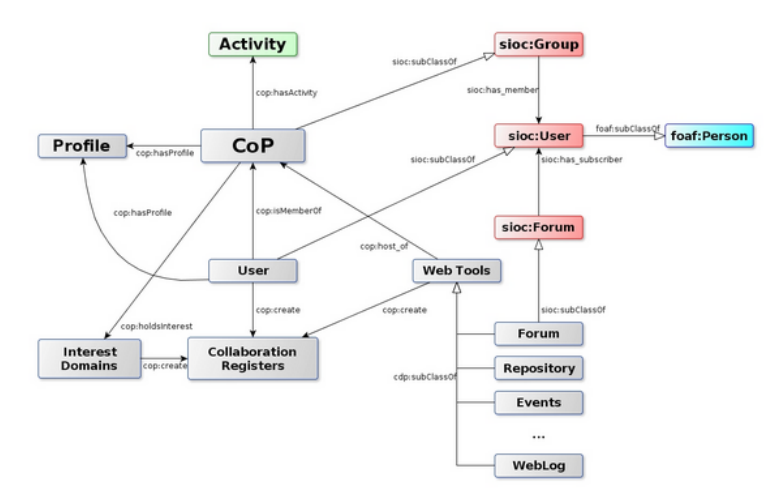

Figura 1. Ontologia de referência representando o Framework CoP [Primo et al. 2012]

relacionamentos que ocorrem entre sujeitos, comunidades e seus conteúdos, através de um modelo ontológico [Ribeiro et al. 2011], [Primo et al. 2012].

O Framework CoP, além de um modelo ontológico, possui uma plataforma de construção de comunidades de prática virtuais. Sua implementação é feita em linguagem Python, usando o Plone como gerenciador de conteúdos e o Zope como o servidor de aplicação. Esta plataforma oferece funcionalidades para o gerenciamento dos participantes, criação de comunidades e criação, compartilhamento e gestão de conteúdos.

\section{Modelo arquitetural dos Registros de Colaboração}

O Framework CoP constitui-se em um modelo ontológico de referência para representação de uma comunidade de prática, incluindo seus participantes, domínio, interações e ferramentas colaborativas [Primo et al. 2012]. O projeto encontra-se em desenvolvimento e não contempla ainda todos os seus componentes descritos no framework ontológico. Em função disso, neste artigo, somente alguns campos já disponíveis na plataforma serão considerados na estrutura dos Registros de Colaboração. Tais campos são descritos na Tabela 1.

Tabela 1. Campos do Registro de Colaboração

\begin{tabular}{|c|c|}
\hline Campo & Conteúdo armazenado \\
\hline id_comunidade & URL da Comunidade \\
\hline id_criador & ID do participante \\
\hline tipo_conteudo & Tipo do conteúdo \\
\hline id_conteudo & URL do conteúdo \\
\hline data_criacao & Data de criação \\
\hline
\end{tabular}

A ideia central do modelo proposto é explorar uma das facetas inerentes a definição de registros de colaboração, para isto, propõe-se a descrição dos mesmos através de triplas em RDF compostas dos conteúdos criados pelos usuários em uma Comunidade de Prática. Esta geração automática é feita utilizando-se o mecanismo de subscribers do Plone. Este mecanismo permite disparar funções associadas a eventos. Ou seja, é perfeitamente possível associar uma função que gera um Registro de Colaboração ao evento lançado pela criação de um tipo de conteúdo qualquer. Um dos pontos que requer 
cuidado nessa abordagem é em relação ao desempenho. Caso essa criação automática seja muito custosa, sua execução de forma síncrona, a cada vez que um conteúdo é criado, pode comprometer o desempenho das Comunidades de Prática. Uma solução para esse caso é a criação de um script assíncrono para a geração dos Registros de Colaboração. Tal script pode ser executado a cada determinado intervalo de tempo, de modo a não comprometer a performance do framework.

A Figura 2 ilustra um grafo representando um exemplo de Registro de Colaboração que segue o modelo proposto. No exemplo, o sujeito registro_colaboracaol do type registro_colaboracao possui um predicado id_comunidade com objeto Comunidade1, um predicado id_criador com objeto Criador, um predicado tipo_conteudo com objeto Link, um predicado id_conteudo com objeto Linkl e um predicado data_criacao com objeto 28/06/2013.

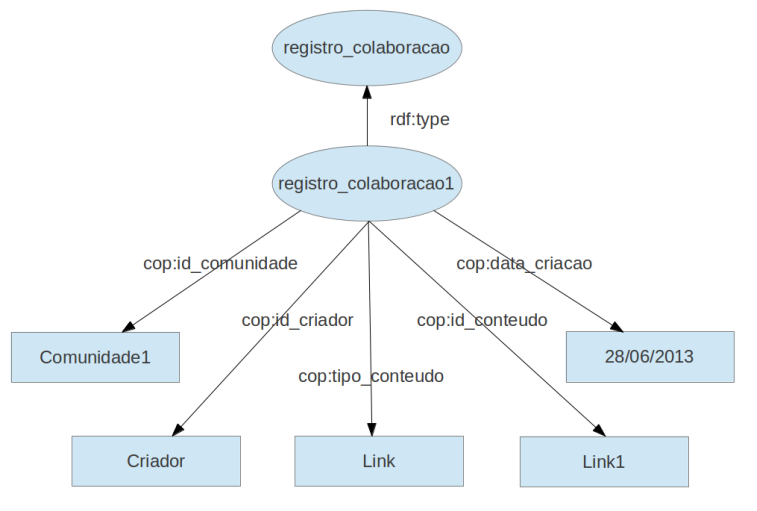

Figura 2. Grafo de um exemplo fictício de Registro de Colaboração

Através destes campos é possível armazenar as principais informações a respeito de um conteúdo compartilhado em uma comunidade de prática, oferecendo conhecimento suficiente para consultas simples. Por exemplo, associando participantes de diferentes comunidades que compartilham os mesmos tipos de conteúdo; ou, associando que tipos de conteúdos caracterizam determinada comunidade; ou, comunidades que compartilham o mesmo domínio, entre outros tipos de consultas.

Os Registros de Colaboração são estruturados seguindo o modelo de dados RDF, segundo a sintaxe RDF/XML, utilizando-se a biblioteca Python $r$ fflib. Esta biblioteca possui diversas funcionalidades que visam o gerenciamento de grafos RDF.

Para a persistência dos dados em formato RDF é utilizado o triple store nativo do framework Sesame. O Sesame foi escolhido pela sua fácil instalação e configuração, além de oferecer uma interface amigável e intuitiva para a consulta dos dados.

O intercâmbio de dados entre o Sesame e o Framework CoP, implementado sobre a pilha Python/Zope/Plone, é feito através do protocolo HTTP (Hypertext Transfer Protocol). Para isso, são utilizadas as bibliotecas httplib e a urllib, padrão da linguagem Python. Estas bibliotecas possuem funcionalidades de alto nível que permitem a comunicação entre serviços que utilizam o protocolo HTTP. A implementação da interface entre triple store e produto através de HTTP ainda torna a solução facilmente portável para outro framework triple store, uma vez que o padrão para comunicação utilizado pela maioria dos frameworks é o protocolo HTTP. 
A última ferramenta a compor esta proposta de solução é o rdfalchemy ${ }^{1}$. Tratase de um ORM (Object RDF Mapper), que é um componente que possibilita fazer o mapeamento dos tipos de recursos RDF em classes Python. A partir desta ferramenta, é modelada a representação RDF para as classes e recursos dos Registros de Colaboração.

A Figura 3 ilustra a arquitetura proposta na solução. A plataforma do Framework CoP disponibiliza uma GUI (Graphical User Interface) para o usuário. O Plone responsabiliza-se pela gestão do conteúdo, enquanto o Zope funciona como servidor de aplicação. Com as bibliotecas Python será desenvolvida a interface de comunicação HTTP entre o framework e o triple store Sesame.

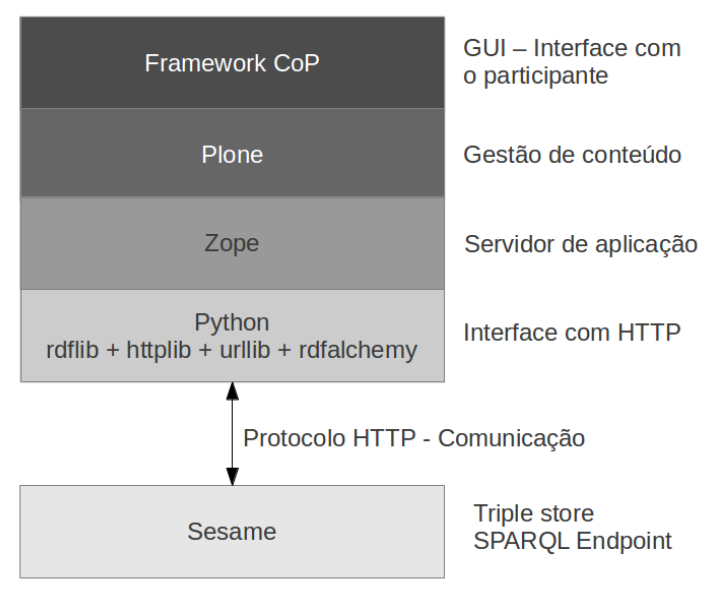

Figura 3. Diagrama da arquitetura da solução

\section{Mapeando interações nos Registros de Colaboração}

A plataforma de Comunidades de Prática que implementa os módulos básicos do CMS Plone foi desenvolvida em módulos. O módulo principal envolve a criação, descrição e desenvolvimento da comunidade de prática com as ferramentas colaborativas, que encontra-se em produção. Para a validação da proposta de representação semântica dos Registros de colaboração, optou-se pelo desenvolvimento de um protótipo que faz um mapeamento de todas as ações colaborativas na plataforma para compor os Registros de Colaboração.

Na prototipação foi criado um produto Plone para que as novas funções semânticas possam ser integradas à implementação do Framework $C o P$. Tal produto foi denominado cop.semantic. O desenvolvimento do protótipo em um produto separado evita a criação de dependência entre o framework e o cop.semantic. Isso possibilita que o framework continue sendo usado de forma independente ao protótipo.

A etapa seguinte no desenvolvimento foi a definição das propriedades referentes aos campos do Registro de Colaboração, em formato RDFS, utilizando-se a sintaxe RDF/XML. A representação das propriedades nesse formato adiciona semântica aos dados que serão gerados em RDF, criando um vocabulário para a criação desses dados.

\footnotetext{
${ }^{1}$ http://www.openvest.com/trac/wiki/RDFAlchemy
} 
Conforme a Figura 4, a classe CollaborationRegister representa um Registro de Colaboração propriamente dito. Já as propriedades community_id, creator_id, content_type, content_id e creation_date representam, respectivamente, a Comunidade de Prática onde foi gerado o registro, o criador do registro, a que tipo de conteúdo o registro se refere, qual o identificador deste registro e a data em que o mesmo foi criado.

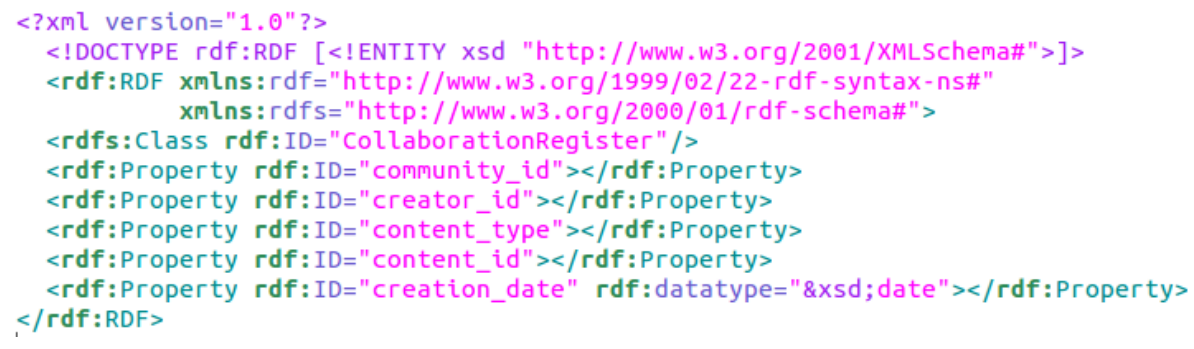

Figura 4. Propriedades do Registro de Colaboração

Após a definição das propriedades dos Registros de Colaboração, foi desenvolvido o script para a geração de tais registros e a persistência dos mesmos na base de dados triple store do Sesame. Em um primeiro momento, a geração dos registros ocorrerá imediatamente após a criação de conteúdos dentro de uma Comunidade de Prática. A Figura 5 apresenta a relação entre a criação de qualquer tipo de conteúdo e a geração de um registro.

Por exemplo: este registro é referente à criação de um conteúdo do tipo "Página" dentro de uma comunidade "Comunidade" por um membro "admin". A URL do conteúdo é armazenada na propriedade content_id; sua data de criação é armazenada na propriedade creation_date; a propriedade content_type refere-se ao tipo de conteúdo, neste caso CoPDocument (página); a URL referente ao criador no portal é armazenada na propriedade creator_id; e a URL da comunidade onde foi criado o conteúdo é armazenada na propriedade community_id.

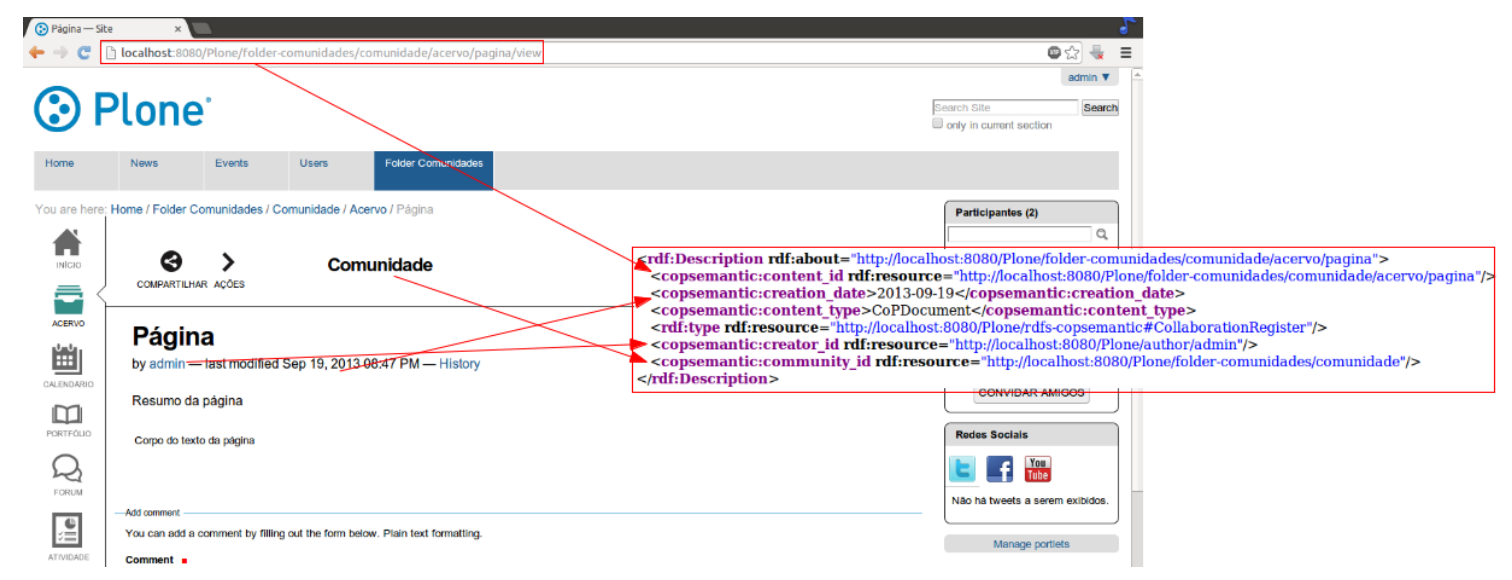

Figura 5. Relação entre o conteúdo e um registro de colaboração em formato RDF

\subsection{Cenários de Uso}

Para a realização dos testes do protótipo foi utilizada uma base de dados real, de um portal com 12 Comunidades de Prática abordando diferentes temas. Este portal possui mais de 
13.000 participantes, e aproximadamente 6.600 conteúdos dos mais diversos tipos criados em sua base de dados.

Dentre todas as ferramentas de interação oferecidas pela plataforma do Framework $\mathrm{CoP}$ a mais utilizada pelos participantes deste portal são os comentários. Este tipo de colaboração pode ser feita tanto nos fóruns de discussão quanto nos próprios conteúdos de outros tipos.

É neste cenário que se desenvolvem experimentos sobre um conjunto de consultas SPARQL. O objetivo destas consultas é a validação da estrutura proposta dos Registros de Colaboração, através da busca de determinados padrões gerados pelo protótipo.

Foram definidos dois cenários principais para testes: consultar as comunidades que tratam sobre determinado tema, assunto ou área do conhecimento e quais são os membros que colaboram através de comentários sobre determinado tema, assunto ou área do conhecimento. Com isto podemos descobrir possíveis intersecções entre que comunidades estão desenvolvendo mais o domínio a que se proporam e futuros algoritmos de recomendação podem direcionar usuários que comentam assuntos cujo domínio pertence a outras comunidades que eles não pertencem.

\subsubsection{Usuários que fazem comentários sobre um mesmo domínio}

Esta consulta visa mostrar quais usuários colaboram através de comentários em Comunidades de Prática que compartilham um mesmo domínio. Estes comentários podem ter sido feitos tanto em conteúdos como em fóruns de discussão. A Figura 6 mostra esta consulta.

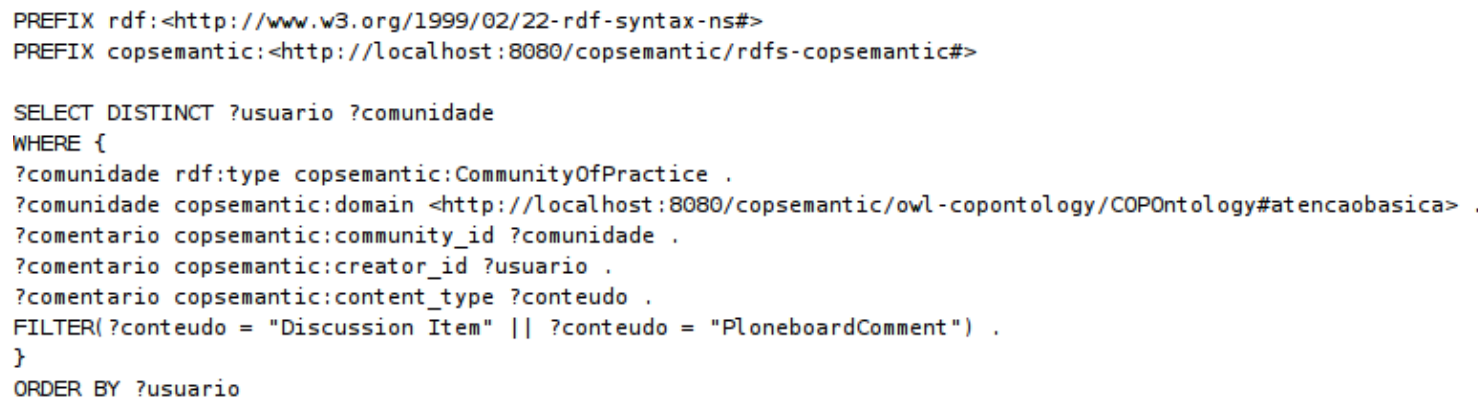

Figura 6. Consulta de usuários que comentam sobre um mesmo domínio

A consulta apresentada faz uma busca dos criadores (copsemantic:creator_id) de conteúdos dos tipos (copsemantic:content_type) "Discussion Item" e "PloneboardComment" (comentários) em todas as Comunidades de Prática (rdf:type copsemantic: CommunityOfPractice) de um determinado domínio (copsemantic:domain), que neste exemplo é "atenção básica". Parte dos resultados desta consulta é ilustrada na Figura 7.

A propriedade copsemantic:domain permite que seja representada a ligação do registro com uma ontologia de domínio. Para os experimentos, foi escrita uma pequena ontologia onde estão definidos alguns indivíduos relacionados a áreas da saúde, tema principal do portal utilizado nos testes. Um trecho desta ontologia é apresentado na Figura 


\begin{tabular}{|c|c|}
\hline Usuario & Comunidade \\
\hline shttp://localhost:8080/dab/author/Rodrigofelberg@gmail.com> & <http://localhost:8080/dab/comunidades/acesso-e-qualidade-na-atencao-basica $>$ \\
\hline <http://localhost:8080/dab/author/TATY IZ@YAHOO.COM.BR > & $\leq$ http://localhost:8080/dab/comunidades/saude-na-escola $>$ \\
\hline <http://localhost:8080/dab/author/acaciosms@gmail.com> & <http://localhost:8080/dab/comunidades/acesso-e-qualidade-na-atencao-basica > \\
\hline <http://localhost:8080/dab/author/acarolns@hotmail.com> & <http://localhost:8080/dab/comunidades/acesso-e-qualidade-na-atencao-basica $>$ \\
\hline <http://localhost:8080/dab/author/addenilda@hotmail.com> & <http://localhost:8080/dab/comunidades/acesso-e-qualidade-na-atencao-basica > \\
\hline shttp://localhost:8080/dab/author/adeiltonmendonca@hotmail.com> & <http://localhost:8080/dab/comunidades/acesso-e-qualidade-na-atencao-basica $>$ \\
\hline shttp://localhost:808o/dab/author/adriana fw@hotmail.com> & 〈http://localhost:8080/dab/comunidades/acesso-e-qualidade-na-atencao-basica $>$ \\
\hline <http://localhost:8080/dab/author/adrianawalherifi@yahoo.com.br> & <http://localhost:8080/dab/comunidades/nasf-nucleo-de-apoio-a-saude-da-familia $>$ \\
\hline <http://localhost:8080/dab/author/adrianoviskpinheiro@hotmail.com> & 〈http://localhost:8080/dab/comunidades/acesso-e-qualidade-na-atencao-basica $>$ \\
\hline <http://localhost:8080/dab/author/adrifeso@bol.com.br> & 〈http://localhost:8080/dab/comunidades/saude-na-escola $>$ \\
\hline <http://localhost:8080/dab/author/adrifeso@bol.com.br> & $\leq$ http://localhost:8080/dab/comunidades/acesso-e-qualidade-na-atencao-basica > \\
\hline <http://localhost:8080/dab/author/ageu1987@hotmail.com> & <http://localhost:8080/dab/comunidades/acesso-e-qualidade-na-atencao-basica > \\
\hline shttp://localhost:808o/dab/author/alana2mc@hotmail.com> & <http://localhost:8080/dab/comunidades/acesso-e-qualidade-na-atencao-basica $>$ \\
\hline
\end{tabular}

Figura 7. Resultados da consulta de usuários que comentam sobre um mesmo domínio

8.

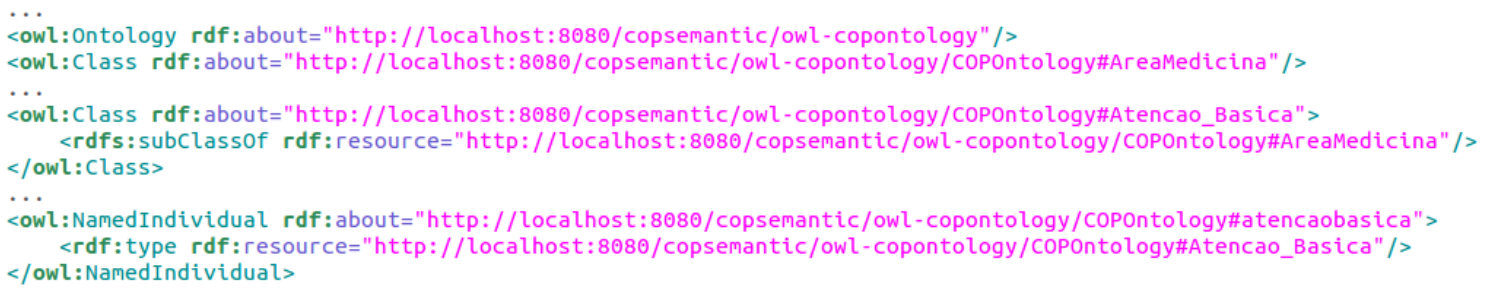

Figura 8. Trechos da ontologia utilizada como referência na base de testes

Apesar de uma nova ontologia ter sido escrita para os testes do protótipo, nada impede que ontologias externas já existentes possam ser referenciadas pela propriedade copsemantic:domain. Esta característica da propriedade permite a integração da solução desenvolvida com outros sistemas já existentes.

\section{Conclusão}

Este artigo apresenta a proposta de desenvolvimento de um dos componentes do Framework CoP seguindo seu modelo ontológico conceitual. Este será um primeiro passo em direção ao desenvolvimento dos outros componentes do framework respeitando este modelo.

A concretização deste componente seguindo as bases teóricas referenciadas ao longo do artigo representará também um avanço significativo no sentido da integração do framework com outras aplicações. A plataforma será elevada ao patamar da Web Semântica, o que abrirá um novo leque de possibilidades referentes ao compartilhamento de informações entre o framework e outras plataformas que seguem modelos semelhantes.

A iminente integração do Framework CoP no mundo da Web Semântica faz emergir uma necessidade que ainda é pouco atendida pelo protótipo já desenvolvido: a utilização de ontologias externas já existentes dentro dos Registros de Colaboração. Esse passo aumentará signigicativamente a integração semântica do framework com o contexto na qual ele está inserido. 


\section{Referências}

Aasman, J. (2011). Will triple stores replace relational databases? Information Management. Disponível em: <http://www.informationmanagement.com/newsletters/database_metadata_unstructured_data_triple_store10020158-1.html> . Acessado em 20/05/2013.

Bandura, A. (1977). Social Learning Theory. Prentice-Hall series in social learning theory. Prentice Hall. ISBN 0138167516.

Berners-Lee, T., Hall, W., and Shadbolt, N. (2006). The semantic web revisited. IEEE Inteligent Systems.

Berners-Lee, T., Hendler, J., and Lassila, O. (2001). The semantic web. Scientific American Magazine, 284(5):28-37.

Bizer, C., Heath, T., and Berners-Lee, T. (2009). Linked Data - The Story So Far.

Brickley, D. and Guha, R. V. (2004). Rdf vocabulary description language 1.0 - rdf schema. Technical report, W3C. Disponível em: <http://www.w3.org/TR/2004/RECrdf-schema-20040210/>. Acessado em 03/05/2013.

Carbonell, J. G. (1970). AI in CAI: An artificial intelligence approach to computer assisted instruction, volume 11. IEEE. IEEE Transactions on Man Machine Systems.

Faye, D. C., Cure, O., and Blin, G. (2012). A survey of rdf storage approaches. ARIMA Journal, 15:11-35. Disponível em: <http://intranet.inria.fr/international/arima/015/ pdf/Vol.15.pp.11-35.pdf > . Acessado em 03/06/2013.

Fiorio, M., da Silva, J. L. T., and Ribeiro, A. M. (2011). Um framework de comunidades de prática em ambientes virtuais de aprendizagem. Revista Novas Tecnologias na Educação.

Hart, J. (2011). Social Learning Handbook. Lulu.com. ISBN 1843101866.

Healy, A. (2009). Communities of Practice as a Support Function for Social Learning in Distance Learning Programs. In M.D. Lytras et al. (Eds.): WSKS 2009, CCIS 49.

Perez, J., Arenas, M., and Gutierrez, C. (2006). Semantics and Complexity of SPARQL. Disponível em: <http://arxiv.org/abs/cs/0605124v1.pdf>. Acessado em 21/03/2013.

Primo, T. T., da Silva, J. L. T., Ribeiro, A. M., Boff, E., and Vicari, R. M. (2012). Towards ontological profiles in communities of practice. IEEE Technology and Engineering Education (ITEE), 7(3):1322. Disponível em: <http://www.ewh.ieee.org/soc/e/sac/itee/index.php/ meem/article/viewFile/223/237>. Acessado em 24/03/2013.

Ribeiro, A. M., da Silva, J. L. T., Boff, E., and Viccari, R. M. (2011). Dos ambientes de aprendizagem às comunidades de prática. $22^{\circ}$ Simpósio Brasileiro de Informática na Educação, pages 690-699.

Sequeda, J. (2013). Introduction to: Triplestores. SemanticWeb.com. Disponível em: <http://semanticweb.com/introduction-to-triplestores_b34996>. Acessado em 19/05/2013. 